\title{
Entrainment of Circadian Rhythms to Temperature Reveals Amplitude Deficits in Fibroblasts from Patients with Bipolar Disorder and Possible Links to Calcium Channels
}

\author{
Victoria Nudell $^{b}$ Heather Wei Caroline Nievergelt $^{a}$, b Adam X. Maihofer ${ }^{b}$ \\ Paul Shilling $^{b}$ Martin Alda ${ }^{c}$ Wade H. Berrettini ${ }^{d}$ Kristen J. Brennand ${ }^{e}$ \\ Joseph R. Calabrese ${ }^{f}$ William H. Coryellg Jonathan M. Covault ${ }^{\text {h }}$ Mark A. Frye ${ }^{\mathrm{i}}$ \\ Fred Gagej Elliot Gershonk Melvin G. McInnis' John I. Nurnbergerm \\ Ketil J. Oedegaard ${ }^{\text {n, o }}$ Tatyana Shekhtman $^{\mathrm{b}}$ Peter P. Zandi ${ }^{\mathrm{p}} \quad$ John R. Kelsoe ${ }^{\mathrm{a}, \mathrm{b}}$ \\ Michael J. McCarthy ${ }^{a, b}$ The Pharmacogenomics of Bipolar Disorder Study \\ aVA San Diego Healthcare System Psychiatry Service, San Diego, CA, USA; ${ }^{b}$ Department of Psychiatry and \\ Center for Circadian Biology, University of California San Diego, La Jolla, CA, USA; ' $D$ Department of Psychiatry, \\ Dalhousie University, Halifax, NS, Canada; 'D Department of Psychiatry, University of Pennsylvania, Philadelphia,

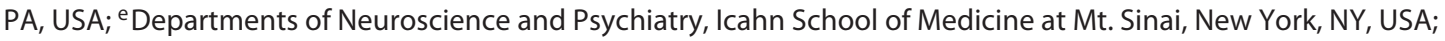 \\ fDepartment of Psychiatry, Case Western Reserve University, Cleveland, OH, USA; 9 Department of Psychiatry, \\ University of lowa, lowa City, IA, USA; ${ }^{h}$ Department of Psychiatry, University of Connecticut, Farmington, CT, USA; \\ 'Department of Psychiatry, Mayo Clinic, Rochester, MN, USA; 'ं Laboratory of Genetics, Salk Institute for Biological \\ Studies, La Jolla, CA, USA; ${ }^{2}$ Department of Psychiatry, University of Chicago, Chicago, IL, USA; 'Department of \\ Psychiatry, University of Michigan, Ann Arbor, MI, USA; 'm Department of Psychiatry, Indiana University, Indianapolis, \\ IN, USA; ${ }^{n}$ Section for Psychiatry, University of Bergen and NORMENT and KG Jebsen Centre for Neuropsychiatry,

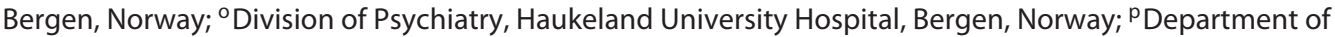 \\ Psychiatry, Johns Hopkins University, Baltimore, MD, USA
}

\section{Keywords}

Bipolar disorder · Circadian rhythm · Calcium channel ·

Gene expression

\begin{abstract}
Bipolar disorder (BD) is characterized by recurrent mood episodes, and circadian rhythm disturbances. Past studies have identified calcium channel genes as risk loci for BD. CACNA1C encodes an L-type calcium channel (LTCC) involved in the entrainment of circadian rhythms to light. Another calcium channel, i.e., the ryanodine receptor (RYR), is involved in
\end{abstract}

(c) 2019 S. Karger AG, Basel

E-Mail karger@karger.com www.karger.com/mnp circadian phase delays. It is unknown whether variants in CACNA1C or other calcium channels contribute to the circadian phenotype in BD. We hypothesized that, by using temperature cycles, we could model circadian entrainment in fibroblasts from $B D$ patients and controls to interrogate the circadian functions of LTCCs. Using Per2-luc, a bioluminescent reporter, we verified that cells entrain to temperature rhythms in vitro. Under constant temperature conditions,

Victoria Nudell and Heather Wei contributed equally to this work.
Michael J. McCarthy

VA San Diego Healthcare System 3350 La Jolla Village Dr. MC116A San Diego, CA 92161 (USA)

E-Mail mmccarthy@ucsd.edu 
the LTCC antagonist verapamil shortened the circadian period, and the RYR antagonist dantrolene lengthened the period. However, neither drug affected temperature entrainment. Fibroblasts from BD patients and controls also entrained to temperature. In cells from BD patients, the rhythm amplitude was lower under entrained, but not constant, conditions. Temperature entrainment was otherwise similar between $\mathrm{BD}$ and control cells. However, the CACNA1C genotype among $B D$ cells predicted the degree to which cells entrained. We conclude that assessment of rhythms under entrained conditions reveals additional rhythm abnormalities in $\mathrm{BD}$ that are not observable under constant temperature conditions.

c 2019 S. Karger AG, Basel

\section{Introduction}

Bipolar disorder (BD) is a severe and debilitating neuropsychiatric illness characterized by recurrent episodes of depression and mania, and altered circadian rhythms in sleep and activity. Family and twin studies suggest that inherited neurobiological factors explain $60-80 \%$ of the variance in $\mathrm{BD}$ [1]. Indeed, recent genome-wide association studies have concluded that $>25 \%$ of the risk for BD can be explained by common genetic variants involving more than 30 genes $[2,3]$. Among the genes linked to BD are several encoding calcium channel subunits, with the L-type calcium channels (LTCC) encoded from CACNA1C being the most strongly implicated. Suggestive, but less conclusive, evidence from genetic and cellular models of BD implicates other genes like CACNA1D, CACNA2D4, and RYR1, which, respectively, encode other LTCC subunits and ryanodine receptors (RYR) $[4,5]$. Therefore, calcium signaling may be critically important to the pathophysiology of BD. However, the channels and the specific functions affected in $\mathrm{BD}$ remain almost entirely unknown.

One role of calcium channels in BD may be the regulation of circadian rhythms. Patients with BD commonly show circadian rhythm disturbances indicative of a misalignment between their internal physiology and external time cues like light [6]. Symptoms of BD include a delayed onset and offset of activity, sleep abnormalities, and a low rhythm amplitude [7-9]. We have shown previously in fibroblasts from $\mathrm{BD}$ patients and controls that BD cells show a variety of circadian rhythm abnormalities. Compared to controls, cells from BD patients have a longer circadian period. Also, whereas in cells from controls lithium increases the amplitude, in cells from BD patients there is a lack of amplitude modulation by lithium [10]. Finally, we have shown that BD patients with an evening chronotype have greater burdens of depressive and manic symptoms and worse therapeutic responses to lithium. Moreover, in cells from lithium-treated BD patients, a longer circadian period, and/or phase delays predict worse treatment outcomes [11]. The attenuation of amplitude in modulation by lithium in BD cells has been linked to LTCC, reduced calcium signaling and genetic variation in CACNA1C and downstream deficits affecting the extracellular signal-regulated kinase (ERK) signal transduction pathway [12, 13]. In the course of this work, it was discovered that CACNA1C is expressed rhythmically in fibroblasts and that, in $\mathrm{BD}$ patients harboring the CACNA1C risk allele rs4765913 (a common T/A SNP), the rhythmic expression of CACNA1C is greatly reduced [13].

LTCC and calcium play an important role in the entrainment of the circadian clock to changes in the light/ dark cycle. Phase delays occur through activation of T-type calcium channels in the early night [14], which subsequently mobilize $\mathrm{Ca}^{2+}$ from intracellular stores through RYR receptor activation [15-17]. Phase advances occur through glutamate signals to the master clock in the suprachiasmatic nucleus (SCN) that activate voltage-dependent LTCC, and subsequent stimulation of protein kinase G [14]. Mutant mice with forebrain selective knockout of Cacnalc expression show normal circadian rhythms under constant dark conditions, but they show a reduced ability to phase advance after a light pulse in the late night [18]. Both phase delay and phase advance pathways converge on the ERK to induce PER1/2 expression [19]. These effects on PER1/2 link calcium signals to the core circadian oscillator, a transcriptional/translational feedback loop made up of the CLOCK/BMAL complex and $\sim 20$ additional "clock genes" that maintain cell autonomous circadian rhythms [20]. The resulting signals advance or delay the start of the circadian cycle to reset phase.

Unlike the SCN, which responds selectively to light, secondary circadian clocks elsewhere in the brain and peripheral tissues respond to a wider range of cues, including steroid hormones, food, and temperature [21]. In this regard, most circadian oscillators in brain areas related to $\mathrm{BD}$ (e.g., the hippocampus and the frontal cortex) are more similar to peripheral cells than to the SCN [6]. By virtue of their less selective responses to environmental cues, non-SCN clocks may be more prone to desynchronization under pathological conditions. Previous work has shown that temperature is a potent stimulus to entrain cellular circadian rhythms in vitro $[21,22]$. This 
finding raises the possibility that circadian entrainment can be studied in vitro in human peripheral cells, possibly allowing a detailed analysis of the circadian mechanisms underlying $\mathrm{BD}$, including calcium channels and other candidate molecules.

Based on these past findings, we attempted to develop an in vitro model of circadian phase advance that would be sensitive to changes in CACNA1C and LTCC function. In this way, we hoped to develop an experimental system in which the effects of genetic perturbations in CACNA1C could be assessed in cells from $\mathrm{BD}$ patients and controls. We hypothesized that calcium channels would contribute to temperature entrainment in cells and that calcium abnormalities in BD would lead to differences in circadian rhythms measured under entrained conditions.

\section{Methods}

\section{Human Subjects}

Skin biopsies were obtained from healthy controls or deidentified patients with BD type I who consented to research while participating in the Pharmacogenomics of Bipolar Disorder (PGBD) trial [23]. The majority of the donors were Caucasian, and most $\mathrm{BD}$ patients were on medication at the time of the biopsy. In order to control for differences in donors' medication history, all cells were passaged at least 4 times before use. Established cell lines were frozen in cryovials and stored in liquid nitrogen until use. BD subjects were evaluated in detail as described previously [23]. The majority of $\mathrm{BD}$ cell line donors (95\%) were of Caucasian ancestry. BD subjects were aged 21-74 years (mean: 46), and males comprised 51\% of the cohort (29/56). Control subjects were evaluated using The Structured Clinical Interview for the DSM-IV to ascertain the psychiatric diagnosis. Subjects with any major psychiatric illnesses, including $\mathrm{BD}$, schizophrenia, major depression, panic, personality disorder, eating disorder, alcohol/drug dependence (other than nicotine), and/or a significant risk for suicide/violence, were excluded. All controls were males of Caucasian ancestry aged 2945 years (mean: 36 ).

\section{Cell Culture}

Human fibroblasts were transduced with the Per2-luc lentiviral reporter gene to assess circadian rhythms as described previously [10]. To facilitate studies with complex experimental designs in a model that approximates human fibroblasts, we developed a mouse fibroblast line that stably expressed the Per2-luc reporter $\left(\mathrm{NIH} 3 \mathrm{~T}^{\mathrm{P} 2 \mathrm{~L}}\right)$, with hygromycin added to the medium as a selection agent [13]. Immortalized mouse hippocampal neurons (mHippoE-18) were purchased from a commercial vendor (Cedarlane) and have been characterized in detail previously [24]. Mouse and human cell lines were grown at $35^{\circ} \mathrm{C}$ to confluence in $100-\mathrm{mm}$ plates in culture media (DMEM with $10 \%$ fetal bovine serum, glutamine $[2 \mathrm{mM}$ ], and antibiotics [penicillin, streptomycin, and amphotericin]).

Cellular Circadian Entrainment and

Calcium Channels

\section{Drugs}

Dantrolene and verapamil were purchased from Tocris Biosciences. The drugs were dissolved in DMSO or sterile water, respectively. Drug concentrations were determined for verapamil based on previously published work [13] and for dantrolene based on the effects on circadian rhythms found in preliminary studies. For all drug studies, identical cell cultures were treated in parallel to allow within-sample matching. Vehicle controls for solvents were used when indicated.

\section{Luminometry}

Luminometers were housed in a dry incubator and maintained in warm room air. Human fibroblast rhythms were recorded using $\sim 1.2 \times 10^{6}$ cells in $35-\mathrm{mm}$ plates over 5 days with a luminometer (Actimetrics). For NIH3T3 ${ }^{\text {P2L }}$ studies, $\sim 2 \times 10^{5}$ cells were dispersed into 24 -well plates and recorded over 5-7 days. Immediately before rhythm recording, the medium was replaced with HEPES-buffered, serum-free recording medium containing $1 \mathrm{mM}$ luciferin (Biosyth International). Dantrolene and verapamil were added to the medium immediately before the beginning of recording and it was left on the cultures for the duration of the 5-7 day recording period.

\section{Temperature Cycle Experiments}

Where indicated, rhythms were measured under temperatureentrained conditions using a 32-well luminometer (Actimetrics) housed inside a programmable incubator (Panasonic MIR-554-PA). Temperature cycle experiments began by allowing samples to stabilize at $35^{\circ} \mathrm{C}$, followed by an increase in temperature to $37.5^{\circ} \mathrm{C}$. The timing was conducted at various repeating intervals of $10-14 \mathrm{~h}$ following a 2-step square wave pattern. All samples were analyzed with 2-4 technical replicates. For $\mathrm{NIH} 3 \mathrm{~T}^{\mathrm{P} 2 \mathrm{~L}}$ cell experiments, parallel cultures grown under identical conditions from the same plate were measured simultaneously in a second luminometer housed in an incubator with the temperature held steady at $35^{\circ} \mathrm{C}$. In this way entrained cells were compared directly to cells maintained under free running conditions. For entrainment studies with human cells, samples were tested first under constant conditions. We tested 56 cell lines from BD patients and 11 cell lines from controls under constant conditions. In some cases, new cultures from the same donors were then subjected to a second round of testing under temperature-entrained conditions at a later time ( $\mathrm{BD}, n=22$; controls, $n=7)$. Since we were unable to match every cell line under both conditions, statistical comparisons between constant and temperature-entrained conditions employed a between group design.

\section{Rhythm Analysis}

Photoemissions (counts per s) from each sample were recorded every 10 min over the duration of the experiment and logged automatically for subsequent analyses. To eliminate artifacts, the first 14-48 h of data were excluded. Background-subtracted luminometry data were fit to a damped sine curve via the least squares method using commercial software (Lumicycle Analysis). Rhythm parameters (period, phase, and amplitude) were estimated for each trace and averaged across replicates. In some experiments, the degree of phase shifting was estimated by calculating the difference between free running and entrained conditions. For analyses of drug effects, 2-way ANOVA with post hoc $t$ tests were conducted separately for each parameter. All analyses were completed using GraphPad Prism version 5.0 (San Diego, CA, USA) with $\alpha<0.05$.

Mol Neuropsychiatry 2019;5:115-124 

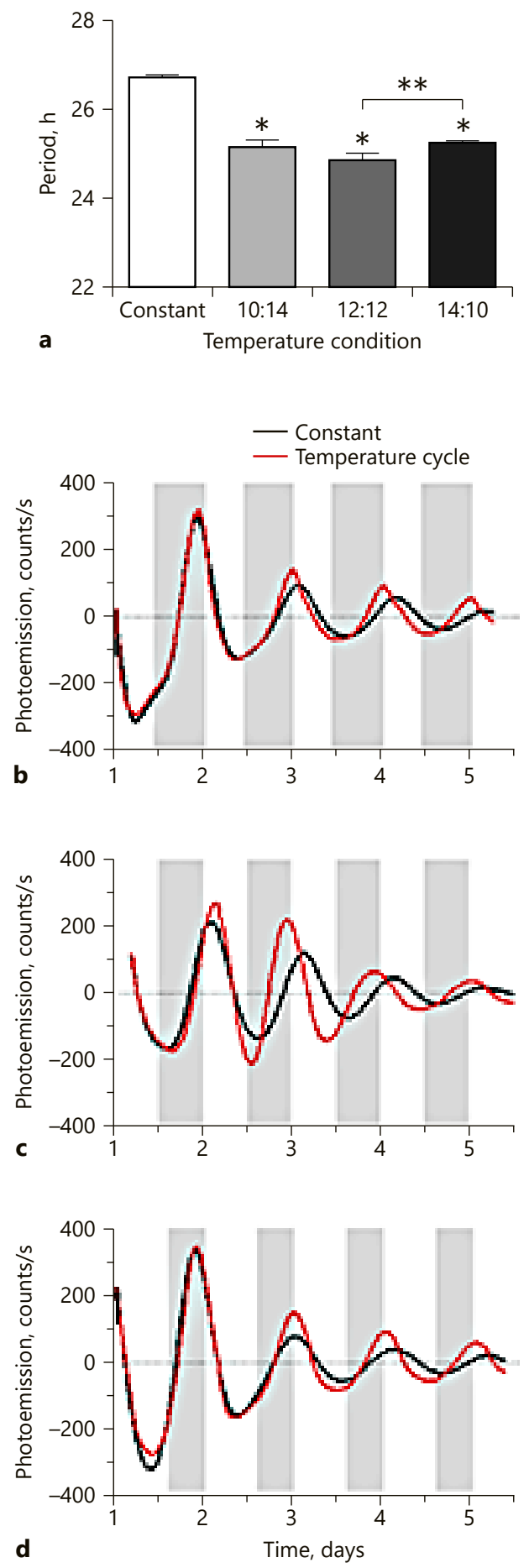

Fig. 1. Temperature cycles entrain rhythms in NIH3T3 cells. a The period is shortened by temperature cycles that approximate the duration of daily light/dark cycles. Compared to cells examined under constant temperature conditions $(n=33)$, cells run under temperature cycles (h at $35^{\circ} \mathrm{C}: \mathrm{h}$ at $37.5^{\circ} \mathrm{C}$ ) of $10: 14(n=3), 12: 12$ $(n=12)$, or 14:10 ( $n=14)$ showed shorter periods of Per2-luc expression that converged upon the period of the temperature cycle. Representative traces are shown for rhythms entrained to 10:14 (b), 12:12 (c), and 14:10 (d) temperature cycles (red) compared to cells from the same plate run in parallel under constant $35^{\circ} \mathrm{C}$ conditions (black). The white bar indicates a temperature of $35^{\circ} \mathrm{C}$, and the gray bars indicate a temperature of $37.5^{\circ} \mathrm{C}$ (applies only to the samples measured under temperature cycles). ${ }^{*}$ Significant (post hoc $t$ test, $p<0.05)$ difference compared to controls. ${ }^{* *}$ Significant difference (post hoc $t$ test, $p<0.05$ ) between 12:12 and 14:10 cycles. 


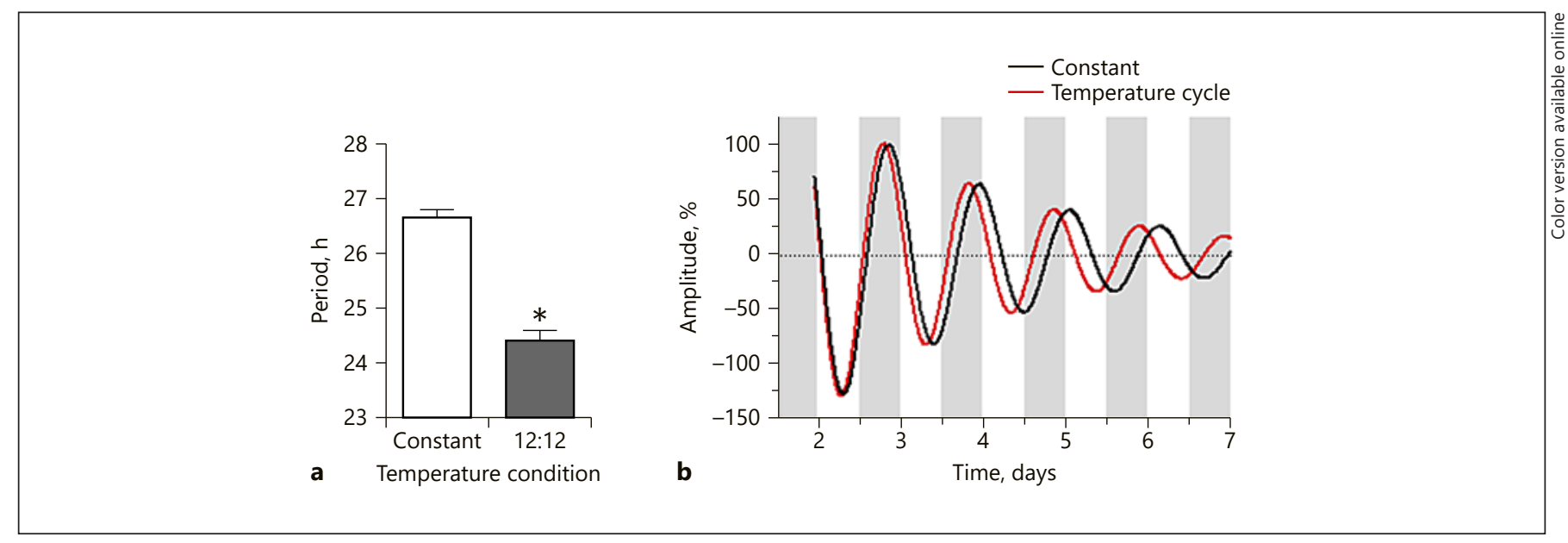

Fig. 2. Temperature cycles entrain rhythms in immortalized mouse hippocampal neurons. a Rhythms in mouse neurons. Compared to neuronal cultures examined under constant temperature conditions $(n=4)$, cells run under a temperature cycle (h at $35^{\circ} \mathrm{C}$ : h at $\left.37.5^{\circ} \mathrm{C}\right)$ of $12: 12(n=4)$ showed shorter periods of Per2-luc expression that converged upon the period of the temperature cycle. Representative traces are shown for rhythms entrained to 12:12 tem- perature cycles (b) (red) compared to neurons run in parallel under constant $35^{\circ} \mathrm{C}$ conditions (black). The white bar indicates a temperature of $35^{\circ} \mathrm{C}$, and the gray bar indicates a temperature of $37.5^{\circ} \mathrm{C}$ (applies only to the samples measured under temperature cycles). ${ }^{*}$ Significant $(p<0.05)$ difference compared to controls as determined by a two-tailed $t$ test.
Similar effects were found in temperature cycles of $10 \mathrm{~h} /$ $14 \mathrm{~h}(10: 14)$ and $14 \mathrm{~h} / 10 \mathrm{~h} \mathrm{(14:10)}$ that approximate the seasonal shifts of the light/dark cycle (Fig. 1a, b, d). The periods of 10:14 and 14:10 did not differ from each other (mean period: $25.2 \pm 0.13$ vs. $25.2 \pm 0.04 \mathrm{~h}$, respectively), but the 12:12 period was significantly shorter than 14:10 (mean period: 24.9 vs. 25.2 h, respectively; Fig. 1a). A single temperature pulse of $37-39^{\circ} \mathrm{C}$ lasting $2-6 \mathrm{~h}$ was insufficient to entrain the cells regardless of the magnitude or timing of the pulse (not shown). To confirm that the temperature cycle mechanism observed in NIH3 $3^{\mathrm{P} 2 \mathrm{~L}}$ cells was also present in neuronal cells, we measured rhythms in immortalized mouse hippocampal neurons. Under constant temperature conditions, the period was $26.6 \mathrm{~h}$. Under 12:12 temperature cycles, the period was significantly shorter $(24.4 \pm 0.2 \mathrm{~h})$, indicating that, as in NIH3T3 ${ }^{\text {P2L }}$ cells, circadian rhythms in mouse neuronal cell lines can entrain to temperature (Fig. 2a, b).

\section{Calcium Channels and Period}

We hypothesized that drugs that target photic entrainment pathways in the SCN would similarly affect the circadian period and the ability of $\mathrm{NIH} 3 \mathrm{~T}^{\mathrm{P} 2 \mathrm{~L}}$ cells to entrain to temperature. Verapamil targets LTCC, which are channels involved in the phase advance response to light. In NIH3 $3^{\mathrm{P} 2 \mathrm{~L}}$ cells, verapamil caused a period-shorten- ing effect (Fig. 3a, b). However, under temperature-entrained conditions, verapamil did not cause any additional period change compared to vehicle-treated cells (Fig. 3c). RYR are involved in the phase delay response. The RYR antagonist dantrolene would therefore be expected to produce the opposite actions of verapamil. Indeed, the application of dantrolene to NIH3T3 ${ }^{\mathrm{P} 2 \mathrm{~L}}$ cells at a constant temperature significantly lengthened the period in a concentration-dependent manner (Fig 3d, e). However, as with LTCC inhibition, the addition of the RYR inhibitor dantrolene did not reverse the periodshortening effect of temperature entrainment (Fig. 3f). These data indicate that blocking calcium channels did affect the circadian period but did not interfere with the ability of $\mathrm{NIH} 3 \mathrm{~T}^{\mathrm{P} 2 \mathrm{~L}}$ cells to entrain to temperature.

\section{Rhythm Parameters under Constant and Entrainment} Conditions in BD Patient and Control Cells

Since rhythms could be altered by temperature cycles, we next investigated this process in human cell lines, including samples from BD patients and controls. As with the mouse fibroblast lines, the circadian period in human fibroblasts could be altered to conform to a temperature cycle. In preliminary studies, cells from the same subjects run on 10:10 and 12:12 cycles showed significantly different periods of 20.9 and $25.4 \mathrm{~h}$, respec- 


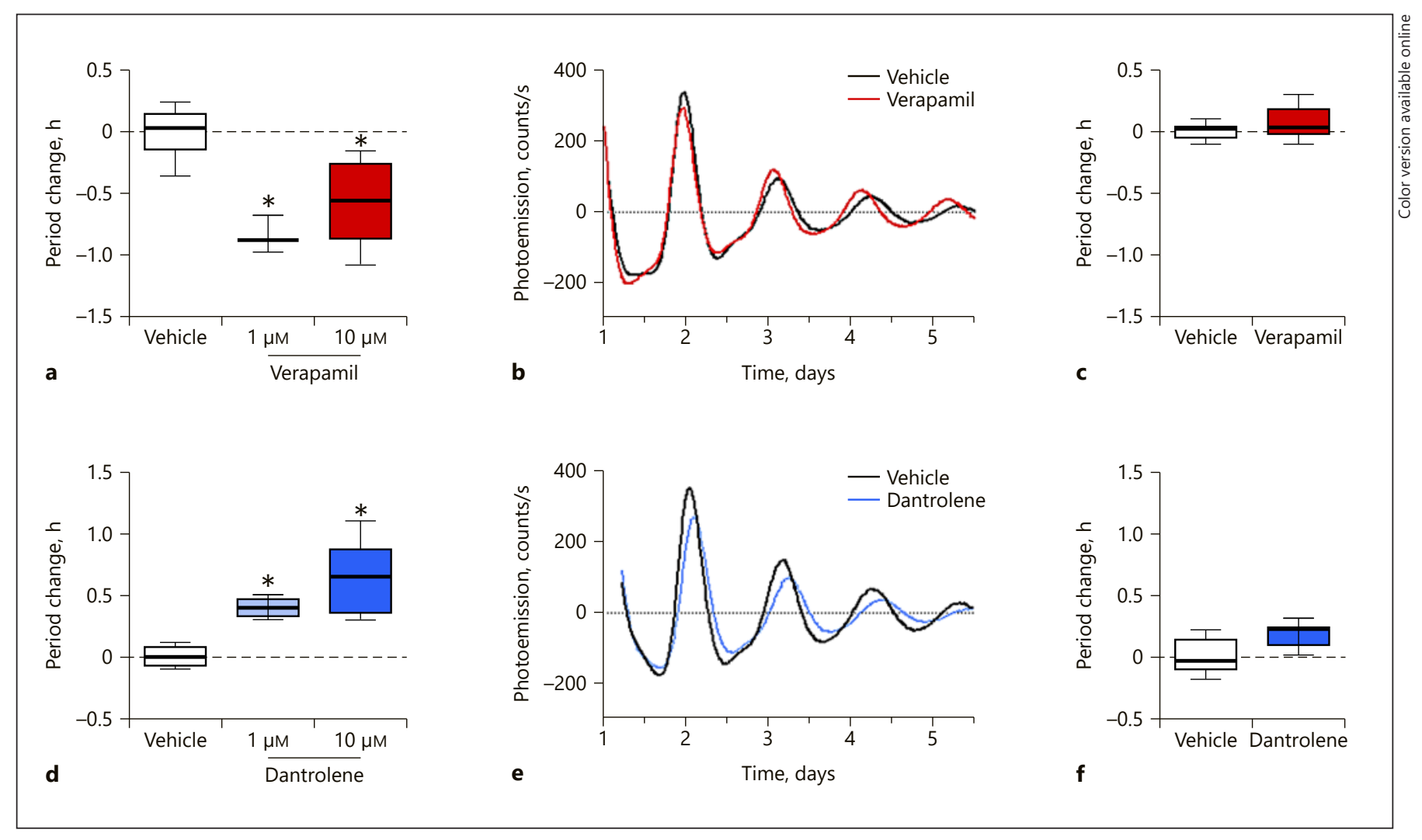

Fig. 3. Calcium channel antagonists alter the circadian period in NIH3T3 cells. a Under constant conditions, the LTCC antagonist verapamil shortens the circadian period at $1(n=3)$ and $10 \mu \mathrm{M}$ $(n=11)$ in NIH3T3 ${ }^{\mathrm{P} 2 \mathrm{~L}}$ cells compared to vehicle-treated controls $(n=22)$. b Representative traces demonstrating the period-shortening effect (under constant conditions) of verapamil $10 \mu \mathrm{M}$ (red) compared to vehicle-treated cells (black) in NIH3 $3^{\mathrm{P} 2 \mathrm{~L}}$ cells. c Under 12:12 temperature-entrained conditions, verapamil at $10 \mu \mathrm{M}$ has no significant effect on period ( $p=0.11, n=9$ /group). $\mathbf{d}$ Under constant conditions, the RYR antagonist dantrolene lengthens the circadian period in a concentration-dependent manner at 1 $(n=4)$ and $10 \mu \mathrm{M}(n=12)$ in NIH3T3 ${ }^{\mathrm{P} 2 \mathrm{~L}}$ cells compared to vehicletreated controls $(n=12)$. e Representative traces demonstrating the period-lengthening effect of dantrolene at $10 \mu \mathrm{M}$ (blue) compared to vehicle-treated cells (black) in NIH3T3 ${ }^{\text {P2L }}$ cells. f Under 12:12 temperature-entrained conditions, dantrolene at $10 \mu \mathrm{M}$ has no significant effect on period ( $n=6$ per group). For each panel, a positive period change indicates a lengthening of period by the drug. Box plots indicate minimum/maximum/mean values and SEM. * Significant $(p<0.05)$ difference compared to controls as determined by one-way ANOVA. tively, indicating that temperature cycles can entrain the circadian period in human cells across a range of several hours (Fig. 4a). Having established that human fibroblasts entrain to temperature, we next set out to measure entrainment to typical environmental conditions using 12:12 temperature cycles in cells from BD patients and controls. We first examined rhythm amplitude. Under constant conditions, there was no significant difference in amplitude between BD patients and controls. However, under entrained conditions, the rhythm amplitude significantly decreased in both groups. Further analyses revealed that under temperature-entrained conditions, the amplitude was significantly higher in control cells compared to BD cells (Fig. 4b, c). We next examined period. Under constant conditions there was no significant difference in period (mean \pm SEM: BD, $25.7 \pm 0.1 \mathrm{~h}$; control, $25.4 \pm 0.3 \mathrm{~h}$ ). Under entrained conditions, the mean period was shortened for all cells. Overall, there was no significant difference in the degree of period shortening between $\mathrm{BD}$ and control cells as a result of the entrainment procedure (Fig. 4c). However, we conducted an exploratory, secondary analysis of the CACNA1C genotype. When examining the data post hoc by CACNA1C genotype, significant differences in period under 12:12 temperature-entrained conditions became apparent, but only in the BD cells (Fig. $4 \mathrm{~d}$, e). In 


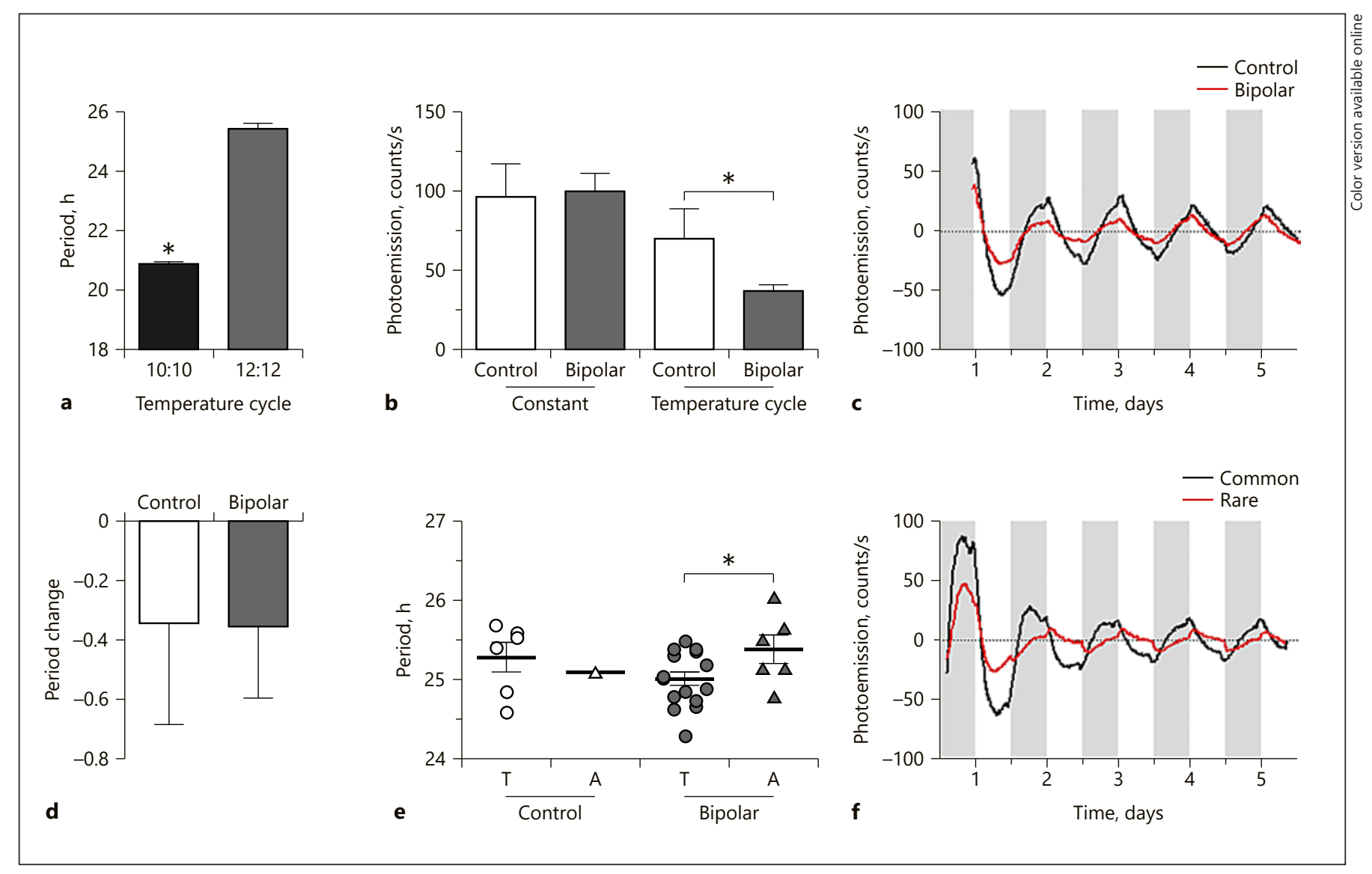

Fig. 4. Entrained circadian rhythms in subjects with $\mathrm{BD}$ and controls. a Entrained period length of human fibroblasts run under varying temperature cycles. b The rhythm amplitude under constant temperature conditions does not differ in fibroblasts from $\mathrm{BD}$ cases and controls, whereas under 12:12 temperature cycle entrained conditions the amplitude is lower in cells from BD patients. The results shown reflect samples collected under constant conditions from controls $(n=10)$ and BD patients $(n=56)$ and samples analyzed under temperature-entrained conditions (BD, $n=22$; controls, $n=7)$. The latter cohort fully includes the former. Twoway ANOVA revealed the main effect of temperature $(p=0.001)$, i.e., and a trend towards a bipolar $\times$ temperature interaction $(p=$ 0.07). A post hoc $t$ test comparing BD and control samples under temperature-entrained conditions revealed a significant difference $(p=0.01)$ (indicated by $*)$. c Representative baseline subtracted, raw data traces of Per2-luc expression rhythms in fibroblast cul-

$\mathrm{BD}$ cells with a CACNA1C risk allele, the mean period was significantly longer compared to $\mathrm{BD}$ cells harboring the common CACNA1C allele ( 25.4 vs. $25.0 \mathrm{~h}$ ), indicating a selective deficit in temperature-induced period shortening in the risk allele carriers. Control cells homozygous for the common CACNA1C allele had a period of $25.3 \mathrm{~h}$, intermediate between the $2 \mathrm{BD}$ groups. tures under temperature entrainment from a control (black) and a $\mathrm{BD}$ patient (red), illustrating the higher rhythm amplitude in controls. White vertical bars indicate the 12 -h periods of $35^{\circ} \mathrm{C}$, and gray bars indicate the 12 -h periods of $37.5^{\circ} \mathrm{C}$. $\mathbf{d}$ Under entrained conditions, there was no significant difference in period in cells from controls vs. BD. However, there was a significant difference in period by genotype. $\mathrm{BD}$ cells harboring a CACNA1C rs4765913 $\mathrm{BD}$ risk allele showed a reduced ability to entrain to $12: 12$ temperature cycles compared to cells homozygous for the more common CACNA1C T allele. ${ }^{*} p<0.05$ in a post hoc $t$ test. e Representative baseline subtracted, raw data traces of Per2-luc expression rhythms in fibroblast cultures from a $C A C N A 1 C$ risk allele carrier (black) and sample homozygous for the CACNA1C common allele (red), illustrating the longer period of the risk allele carrier under entrained conditions. White bars indicate the 12 -h periods of $35^{\circ} \mathrm{C}$, and gray bars indicate the 12 -h periods of $37.5^{\circ} \mathrm{C}$.
Only a single control subject was a CACNA $1 C$ risk allele carrier (Fig. 4e). These preliminary results indicate that $\mathrm{BD}$ cells harboring the CACNA1C risk allele may not respond as robustly to the environmental inputs underlying circadian entrainment as do cells homozygous for the common CACNA $1 C$ allele. There was no significant effect of the CACNA1C genotype on amplitude. 


\section{Discussion}

Past work implicates $C A C N A 1 C$ as a risk-associated gene in $\mathrm{BD}$. Given that the protein products of $C A C N A 1 C$ (Cav1.2 calcium channels) are suitable drug targets and very well characterized, understanding the role of CACNA $1 C$ and calcium signaling in $\mathrm{BD}$ is a high priority in translational research. However, the functional implication of genetic variation in CACNA1C in BD remain unknown. Presently, there are no validated in vitro assays that can interrogate Cav1.2 function in $\mathrm{BD}$ in a manner that unambiguously maps onto a relevant clinical phenotype. The development of a sensitive assay that discriminates cells from $\mathrm{BD}$ and unaffected samples would be a valuable advance for therapeutic drug screening and/or biomarker development. With this framework in mind, we examined whether circadian phase shifting could be used to assess the function of calcium channels in vitro, both in mouse and in human fibroblast cultures. In so doing, we also hypothesized that some of the circadian rhythm abnormalities observed in BD may be explained by genetic variations in CACNA1C. Our main finding is that amplitude abnormalities in circadian rhythms are revealed selectively by assessing the rhythm under entrained conditions. We did find that calcium channels regulate rhythms in peripheral cells but are not critical for temperature entrainment. With respect to entrainment deficits, we found no major effect of BD in our case-control analysis but we did find suggestive evidence of entrainment deficits in a subset of $\mathrm{BD}$ samples harboring the CACNA1C risk allele in exploratory analyses. This latter finding should be considered preliminary and is in need of replication in a larger sample. Our work has the strengths that we are able to study a disease-relevant mechanism in human BD patient samples and we were able to measure rhythms prospectively for several days in high resolution while modelling entrainment in vitro. Limitations of our work include the relatively small sample size of cell line donors. Due to the cost and technical challenges of running large numbers of human samples (that require viral transfection of each sample), we conducted the majority of our mechanistic studies in animal cells (that can be stably transfected) and therefore we cannot be certain that the mechanisms we identified in mouse cells apply fully to human cells.

As others have described previously [21,22], we found that temperature cycles were able to entrain fibroblast circadian rhythms. Under entrained conditions, the period was shortened to more closely approximate the temperature cycle. We found previously that cells from $\mathrm{BD}$ pa- tients have longer periods than controls [10] and that, in cells from BD patients who were nonresponsive to lithium treatment, the period is longer compared to cells from lithium responders [11]. Therefore, we hypothesized that these period differences may translate into impaired temperature entrainment in $\mathrm{BD}$ cells compared to control cells. However, this was not the case. Cells from BD and control donors showed similar period lengths under entrained conditions.

In contrast to period, we did find amplitude differences in the cells from BD donors. Selectively under entrained conditions, the rhythm amplitude was lower in $\mathrm{BD}$ cells compared to control cells. The same samples run under constant conditions did not show any amplitude abnormality, indicating that measurement of rhythms under entrained conditions could possibly reveal rhythm abnormalities that were not detectable previously under constant temperature ("free running") conditions. We have examined rhythm amplitude under constant conditions previously and found a similar lack of a difference between $\mathrm{BD}$ cases and controls under constant conditions [10]. However, as in the present study where temperature entrainment revealed a selective amplitude difference, we found in the previous study amplitude differences that were apparent only after treating the cells with lithium. Our amplitude and entrainment findings may be linked. It has been reported previously that low-amplitude rhythms entrain to temperature stimuli more readily than high-amplitude rhythms [25]. In this way, the lower-amplitude rhythms in BD cells under temperature cycles may shorten the period more readily. This more flexible rhythm may offset any potential entrainment deficit stemming from the longer period we previously found in BD cells.

While we did not examine it directly in the present study, it is possible that lithium and temperature entrainment engage similar signal transduction pathways to relay inputs to the circadian clock. We reported previously that the $\mathrm{BD}$-associated differences in amplitude under lithium-treated conditions involved calcium [13] and ERK signaling [12], pathways involved in photic entrainment in the SCN $[14,15,18,19]$. The relative decrease in amplitude in the BD samples under both lithium treatment and entrained conditions may imply a loss of function in one or more of these pathways in BD. Additional work is required to dissect this putative overlap in more detail, particularly with regard to ERK, which was not evaluated under temperature-entrained conditions. In the present study, we found that pharmacological antagonism of calcium channels had effects on 
the circadian period. The LTCC antagonist verapamil shortened the period, while the RYR antagonist dantrolene lengthened it. However, the lack of an effect of these drugs on entrainment suggests that they are unlikely to be the primary mechanism underlying the finding of a BD-associated low amplitude under entrained conditions that we report presently. Our current data suggest that the effects of calcium and temperature on rhythms are nonadditive and that the effects of temperature on rhythms dominate any input from the 2 calcium pathways we examined. Nonetheless, we observed in $\mathrm{BD}$ samples carrying the $C A C N A 1 C \mathrm{BD}$ risk allele that period shortening under a temperature cycle was less robust than in cells carrying the common allele. This finding is similar to the effect we reported previously in which carriers of the CACNA1C risk allele demonstrated a reduced amplitude after treatment with lithium [13]. Taken together, we conclude that the entrainment pathway and lithium may engage similar pathways that, if understood, could elucidate more about lithium's therapeutic mechanism and/or the pathophysiological mechanisms underlying BD.

\section{Acknowledgement}

The authors wish to thank Drs. David Welsh, Alessandra Por$\mathrm{cu}$, and Himanshu Mishra, who provided useful feedback on early versions of this work, and the patients who contributed samples and time to support this research activity.

\section{Statement of Ethics}

The use of materials from human subjects was conducted in accordance with all pertinent laws and regulations. Deidentified samples were shared with the principal investigator (M.J.M.) as allowed by the subjects' original informed consent documents. The research protocol was reviewed and approved by the VASDHS IRB and the individual sites that contributed samples.

\section{Disclosure Statement}

The authors have no conflicts of interests to declare.

\section{Funding Sources}

This work was funded by an International Bipolar Disorder Foundation Catalysts Award to M.J.M. and a VA Merit Award (VA BLR\&D BX003431, principal investigator: MJM). Sample collection from the PGBD trial was supported by NIMH/NIGMS (MH92758) and the Canadian Institutes of Health Research (No. 64410).

\section{Author Contributions}

V.N. and H.W. conducted experiments, analyzed data, and wrote sections of this paper. K.J.B., T.S., and F.G. established patient cell lines and J.M.C. established control cell lines. C.N., A.X.M., and P.S. provided data management. M.A., W.H.B., J.R.C., M.A.F., J.M.C., E.G., M.G.M., J.I.N., K.J.O., P.P.Z., and J.R.K. were PGBD investigators and/or recruited subjects from which cell cultures were established. M.J.M. designed the project, analyzed data, and wrote this paper.

\section{References}

1 McGuffin P, Rijsdijk F, Andrew M, Sham P, Katz R, Cardno A. The heritability of bipolar affective disorder and the genetic relationship to unipolar depression. Arch Gen Psychiatry. 2003 May;60(5):497-502.

2 Psychiatric GWAS Consortium Bipolar Disorder Working Group. Large-scale genomewide association analysis of bipolar disorder identifies a new susceptibility locus near ODZ4. Nat Genet. 2011 Sep;43(10):977-83.

3 Cross-Disorder Group of the Psychiatric Genomics Consortium. Identification of risk loci with shared effects on five major psychiatric disorders: a genome-wide analysis. Lancet. 2013 Apr;381(9875):1371-9.

4 Mertens J, Wang QW, Kim Y, Yu DX, Pham S, Yang B, et al.; Pharmacogenomics of Bipolar Disorder Study. Differential responses to lithium in hyperexcitable neurons from patients with bipolar disorder. Nature. 2015 Nov;527(7576):95-9.
5 Nurnberger JI Jr, Koller DL, Jung J, Edenberg HJ, Foroud T, Guella I, et al.; Psychiatric Genomics Consortium Bipolar Group. Identification of pathways for bipolar disorder: a metaanalysis. JAMA Psychiatry. 2014 Jun;71(6): 657-64.

6 McCarthy MJ, Welsh DK. Cellular circadian clocks in mood disorders. J Biol Rhythms. 2012 Oct;27(5):339-52.

7 Harvey AG. Sleep and circadian rhythms in bipolar disorder: seeking synchrony, harmony, and regulation. Am J Psychiatry. 2008 Jul; 165(7):820-9.

8 Pagani L, St Clair PA, Teshiba TM, Service SK, Fears SC, Araya C, et al. Genetic contributions to circadian activity rhythm and sleep pattern phenotypes in pedigrees segregating for severe bipolar disorder. Proc Natl Acad Sci USA. 2016 Feb;113(6):E754-61.
9 Gonzalez R, Tamminga CA, Tohen M, Suppes $T$. The relationship between affective state and the rhythmicity of activity in bipolar disorder. J Clin Psychiatry. 2014 Apr;75(4):e317-22.

10 McCarthy MJ, Wei H, Marnoy Z, Darvish RM, McPhie DL, Cohen BM, et al. Genetic and clinical factors predict lithium's effects on PER2 gene expression rhythms in cells from bipolar disorder patients. Transl Psychiatry. 2013 Oct;3(10):e318.

11 McCarthy M, Wei H, Nievergelt C, Stautland A, Maihofer AX, Welsh DK, et al.: Chronotype and cellular circadian rhythms predict the clinical response to lithium maintenance treatment in patients with bipolar disorder. Neuropsychopharmacology. 2019 Feb;44(3):620-8.

12 McCarthy MJ, Wei H, Landgraf D, Le Roux MJ, Welsh DK. Disinhibition of the extracellular-signal-regulated kinase restores the amplification of circadian rhythms by lithium in cells from bipolar disorder patients. Eur Neuropsychopharmacol. 2016 Aug;26(8):1310-9. 
13 McCarthy MJ, LeRoux M, Wei H, Beesley S, Kelsoe JR, Welsh DK. Calcium channel genes associated with bipolar disorder modulate lithium's amplification of circadian rhythms. Neuropharmacology. 2016 Feb;101:439-48.

14 Kim DY, Choi HJ, Kim JS, Kim YS, Jeong DU, Shin HC, et al. Voltage-gated calcium channels play crucial roles in the glutamate-induced phase shifts of the rat suprachiasmatic circadian clock. Eur J Neurosci. 2005 Mar; 21(5):1215-22.

15 Pfeffer M, Müller CM, Mordel J, Meissl H, Ansari N, Deller T, et al. The mammalian molecular clockwork controls rhythmic expression of its own input pathway components. J Neurosci. 2009 May;29(19):6114-23.

16 Gamble KL, Ciarleglio CM. Ryanodine receptors are regulated by the circadian clock and implicated in gating photic entrainment. J Neurosci. 2009 Sep;29(38):11717-9.
17 Ding JM, Buchanan GF, Tischkau SA, Chen D, Kuriashkina L, Faiman LE, et al. A neuronal ryanodine receptor mediates light-induced phase delays of the circadian clock. Nature. 1998 Jul;394(6691):381-4.

18 Schmutz I, Chavan R, Ripperger JA, Maywood ES, Langwieser N, Jurik A, et al. A specific role for the REV-ERB $\alpha$-controlled LType Voltage-Gated Calcium Channel CaV1.2 in resetting the circadian clock in the late night. J Biol Rhythms. 2014 Aug;29(4): 288-98.

19 Obrietan K, Impey S, Storm DR. Light and circadian rhythmicity regulate MAP kinase activation in the suprachiasmatic nuclei. Nat Neurosci. 1998 Dec;1(8):693-700.

20 Partch CL, Green CB, Takahashi JS. Molecular architecture of the mammalian circadian clock. Trends Cell Biol. 2014 Feb;24(2):90-9.

21 Brown SA, Zumbrunn G, Fleury-Olela F, Preitner N, Schibler U. Rhythms of mammalian body temperature can sustain peripheral circadian clocks. Curr Biol. 2002 Sep;12(18): 1574-83.
22 Buhr ED, Yoo SH, Takahashi JS. Temperature as a universal resetting cue for mammalian circadian oscillators. Science. 2010 Oct; 330(6002):379-85.

23 Oedegaard KJ, Alda M, Anand A, Andreassen OA, Balaraman Y, Berrettini WH, et al. The Pharmacogenomics of Bipolar Disorder study (PGBD): identification of genes for lithium response in a prospective sample. BMC Psychiatry. 2016 May;16(1):129.

24 Gingerich S, Kim GL, Chalmers JA, Koletar MM, Wang X, Wang Y, et al. Estrogen receptor $\alpha$ and G-protein coupled receptor 30 mediate the neuroprotective effects of $17 \beta$-estradiol in novel murine hippocampal cell models. Neuroscience. 2010 Sep;170(1):54-66.

25 Barrett RK, Takahashi JS. Lability of circadian pacemaker amplitude in chick pineal cells: a temperature-dependent process. J Biol Rhythms. 1997 Aug;12(4):309-18. 